\title{
Developmental anomalies in Drosophila hybrids are apparently caused by loss of microchromosome
}

\author{
H. Allen Orr
}

Department of Ecology and Evolution, University of Chicago, 1103 E. 57th Street, Chicago, IL 60637, U.S.A.

Hybrids produced by crossing Drosophila virilis females to $D$. lummei males suffer from many developmental anomalies; the reciprocal hybridization yields normal offspring. Genetic analysis reveals that these anomalies involve a maternal effect: whether or not an individual will show an anomaly depends upon his mother's nuclear genotype. Several lines of evidence suggest that the proximal cause of the anomalies is the elimination of the $D$. lummei microchromosome (chromosome 6) from hybrids. Loss of the $D$. lummei microchromosome in this hybridization is known to involve a maternal effect (Evgen'ev, 1973), as mitosis in early development is under the control of maternally-acting genes.

\section{INTRODUCTION}

Developmental and morphological anomalies are common among species hybrids. Drosophila melanogaster-D. simulans hybrids, for example, often suffer from missing bristles and incomplete sclerotization of the abdomen (Sturtevant, 1920, 1929). Unfortunately, almost nothing is known about the genetic causes of such hybrid anomalies.

Elucidation of the basis of these anomalies may throw some light on speciation. In particular, it will be useful to determine whether the genetic basis of developmental anomalies more closely resembles the basis of hybrid inviability/sterility or that of normal morphological differences between species. We know that the former phenomena affect hybrid males more than females (Haldane's rule), involve a large effect of the $\mathrm{X}$ chromosome (Coyne and Orr, 1989), and often show maternal effects (Orr, 1989). The genes underlying morphological differences between species, however, do not map disproportionately to the X (see Coyne and Orr, 1989), and do not seem to involve frequent maternal effects. As Coyne and Orr (1989) note, certain explanations of these different patterns predict that morphological/developmental anomalies in hybrids will behave like postzygotic isolation (e.g., obey Haldane's rule and show a large effect of the X), while other explanations predict that the "genetic architecture" of morphological anomalies will resemble the architecture of normal morphological differences between species.

During a study of the basis of postzygotic isolation in the virilis group of Drosophila (Orr and Coyne, 1989), we noticed that hybrids between $D$. virilis and $D$. lummei frequently showed several morphological anomalies. The following anomalies were observed: greatly reduced eye resembling the "eyeless" mutation of $D$. melanogaster (usually only one eye affected), unequal wing lengths, broken or incomplete wing veins, twisted abdomen, and missing or reduced thoracic bristles. The first three anomalies were particularly common. Anomalies only appeared when $D$. virilis females were crossed to $D$. lummei males; the reciprocal cross yielded normal progeny. This result was previously reported by Sokolov $(1948,1959)$ (although he misidentified $D$. lummei as "D. littoralis" (Throckmorton, 1982)). As the D. lummei strains that Sokolov found to produce the most frequent anomalies have been lost, little additional work on these anomalies has been undertaken (however, as this paper goes to press, an abstract by Heikkinen and Lumme (1989) confirms the non-reciprocal nature of these anomalies; see below). Fortunately, the $D$. lummei strain we possessed produced frequent anomalies when hybridized with $D$. virilis. Also, fortunately, male and female hybrids between $D$. 
virilis and D. lummei are viable and fertile, allowing genetic analysis of the basis of these anomalies. I report the results of this analysis here.

\section{MATERIALS AND METHODS}

The following $D$. virilis and $D$. lummei stocks were used: $D$. virilis Pasadena, D. virilis white ( $w: 1-105$ [all map positions from Alexander (1976)]), D. virilis peach; glossy ( pe: $5-203 ; g l: 6-1 \cdot 0)$, D. virilis varnished (va 2-231.5), D. virilis broken; tiny bristles, gap; cardinal; peach (b 2-188.0; tb 3104.0, gp 3-118.0; cd 4-32.2; pe) and D. lummei Finland. A few crosses involving $D$. americana Red Cloud, D. novamexicana San Antonio, and $D$. texana Morrilton were also made.

The method used to determine the genetic basis of the hybrid anomalies is similar to that used by Orr and Coyne (1989) to analyse the basis of hybrid sterility in the $D$. virilis group: $D$. virilis flies carrying morphological markers are hybridized with wild-type $D$. lummei flies, and the fertile $F_{1}$ hybrids are backcrossed to either parental species. By correlating the presence of hybrid anomalies with particular markers, one can determine which chromosomes play a large role in the production of the anomalies. In most cases only the X chromosome carried a marker, allowing one to separate the effects of the $X$ from that of the unmarked autosomes. The following anomalies were scored: (1) reduced eye ("eyeless"); (2) obvious wing length asymmetries; (3) wing vein anomalies (usually broken or missing veins; more rarely, small extra vein-segments appeared and were included in this category).

As preliminary results demonstrated that the D. virilis cytoplasm plays a large role in the production of anomalies, most crosses were designed to determine whether this effect results from a maternal effect (i.e., a mother's nuclear genotype determining her progeny's phenotype) or from a true cytoplasmic effect (e.g., an endosymbiont or mitochondrial gene). The role of an endosymbiont was tested by rearing flies on tetracycline, following the protocol of Hoffman and Turelli (1988).

Unless otherwise indicated, all crosses were made for one week at $18^{\circ} \mathrm{C}$ and then transferred to fresh vials at $22^{\circ} \mathrm{C}$.

\section{RESULTS}

Individuals from the pure species stocks of $D$. virilis and $D$. lummei show no developmental anomalies (table 1). However, the $D$. virilis $w$ female $\times D$. lummei male hybridization produces males and females who frequently suffer developmental anomalies (table 1). Hybrid females and males are affected at the same rate $(27 \cdot 8$ vs. $26 \cdot 1$

Table 1 Number of individuals showing various developmental anomalies in pure species, $F_{1}$ hybrids, and hybrid backcrosses. In this and all subsequent tables, $\mathrm{V}=D$. virilis and $\mathrm{L}=D$. lummei. In a cross, the species origin of the maternal species is given first, followed by the paternal species, e.g., $\mathrm{VL}=D$. virilis $q \times D$. lummei o. $(\mathrm{VL}) \mathrm{L}=\mathrm{F}_{1} \uparrow\left(D\right.$. virilis $\$ \times D$. lummei $\left.{ }^{\star}\right) \times D$. lummei ${ }^{\star}$

\begin{tabular}{|c|c|c|c|c|c|}
\hline Genotype & Eyeless & $\begin{array}{l}\text { Winglength } \\
\text { asymmetry }\end{array}$ & $\begin{array}{l}\text { Wing vein } \\
\text { anomaly }\end{array}$ & Normal & Total \\
\hline \multicolumn{6}{|c|}{ D. virilis $w$} \\
\hline$q$ & 0 & 0 & 0 & 200 & 200 \\
\hline o & 0 & 0 & 0 & 200 & 200 \\
\hline \multicolumn{6}{|c|}{ D. lummei Finland } \\
\hline$q$ & 0 & 0 & 0 & 195 & 195 \\
\hline$\delta$ & 0 & 0 & 0 & 211 & 211 \\
\hline \multicolumn{6}{|l|}{ VL } \\
\hline$q$ & 17 & 38 & 24 & 205 & 284 \\
\hline$\sigma$ & 16 & 23 & 18 & 161 & 218 \\
\hline \multicolumn{6}{|l|}{ LV } \\
\hline$q$ & 0 & 1 & 0 & 327 & 328 \\
\hline$\delta$ & 0 & 0 & 0 & 325 & 325 \\
\hline \multicolumn{6}{|l|}{$(\mathrm{VL}) \mathrm{L}$} \\
\hline q & 0 & 0 & 13 & 358 & 371 \\
\hline$w \delta$ & 0 & 2 & 1 & 102 & 105 \\
\hline$+\sigma^{*}$ & 0 & 1 & 2 & 152 & 155 \\
\hline
\end{tabular}


per cent anomalous, respectively; $\chi^{2}=1 \cdot 21, \mathrm{df}=3$, $P>0.75)$. Therefore, the hybrid anomalies do not obey Haldane's rule.

The reciprocal cross does not produce anomalous progeny (table 1). The difference between the frequency of anomalies in the two reciprocal hybridizations is highly significant (for females, $\chi^{2}=101 \cdot 44$, $\mathrm{df}=3, P<0 \cdot 0001$; for males, $\left.\chi^{2}=94.94, \mathrm{df}=3, P<0.0001\right)$. Because the $\mathrm{F}_{1}$ females from the two reciprocal hybridizations possess the same nuclear genotype, production of the developmental anomalies must involve the cytoplasm. There are two ways this can occur: production of anomalies may depend upon the nuclear genotype of a hybrid's mother (a "maternal effect"), or may involve some autonomous cytoplasmic agent, e.g., a mitochondrial gene or an endosymbiont to which $D$. virilis is resistant (a "cytoplasmic effect").

To test for the presence of an endosymbiont, the $D$. virilis $w$ female $\times D$. lummei male hybridization was performed, and progeny reared, on medium containing tetracycline. Control crosses were run on medium lacking tetracycline. Tetracycline treatment did not cure the hybrid anomalies (table 2). Indeed, more anomalies appeared among hybrids receiving the tetracycline treatment $\left(\chi^{2}=\right.$ $54 \cdot 73$, df $=3, P<0 \cdot 0001)$. While this result does not rule out the endosymbiont-hypothesis (a tetracycline-insensitive microorganism could be involved), it suggests that it is worthwhile to test for maternal effects.

To determine whether the anomalies depend on maternal genotype or merely on the presence of $D$. virilis cytoplasm, I crossed hybrid $F_{1}$ females carrying $D$. virilis cytoplasm to $D$. lummei males. Table 1 shows that $F_{1}$ females produce far fewer anomalous progeny than do pure $D$. virilis females crossed to the same males ( 3 vs. 25 per cent, respectively; pooling homogeneous female and male data, $\left.\chi^{2}=63 \cdot 45, \mathrm{df}=3, P<0 \cdot 0001\right)$. This suggests that a mitochondrial factor or endosymbiont is not involved: although these backcross hybrids carry mitochondria and any endosymbionts from $D$. virilis on a largely $D$. lummei genetic background, they show a greatly reduced frequency of anomalies. This result instead suggests that the anomalies involve a maternal effect: these backcross hybrids show few anomalies because their mothers had an $F_{1}$ hybrid, not a pure species, genotype. If a maternal effect is involved, the maternally-acting genes from $D$. virilis are nearly recessive: $D$. virilis $m_{\text {virilis }} / m_{\text {virilis }}$ females produce many anomalous offspring, while $M_{\text {lummei }} / m_{\text {viritis }}$ $\mathrm{F}_{1}$ hybrid females produce very few.

Proof that maternal genotype-not the species source of cytoplasm-causes the anomalies requires showing that hybrids carrying cytoplasm from the "wrong" species, i.e., D. lummei, can also suffer anomalies. As the above results suggest that hybrid anomalies are most common when mothers are homozygous for some putative maternallyacting allele from $D$. virilis, I backcrossed $F_{1}$ females from a $D$. lummei female $\times D$. virilis $w$ male cross to $D$. virilis $w$ males. The resulting backcross females carry cytoplasm from $D$. lum$m e i$, but, on average, are homozygous at 50 per cent of their loci for alleles from $D$. virilis (they are $D$. virilis/D. lummei heterozygotes at their remaining loci).

The results are unambiguous: these hybrid females $d o$ produce anomalous offspring when crossed to $D$. lummei males, despite the fact that their cytoplasm ultimately derives from $D$. lummei (table 3 ). This proves that hybrid anomalies do not result from an endosymbiont or mitochondrial gene, but from a maternal effect. The data also suggest that the maternally-acting genes are not $\mathrm{X}$-linked. As the maternally-acting alleles from $D$. virilis must be nearly recessive, white $\left(X_{\text {virilis }} / X_{\text {virilis }}\right)$ backcross females should produce more anomalous progeny than wild-type $\left(X_{\text {virilis }} / X_{\text {lummei }}\right)$ females if the genes are $X$-linked. In fact, these females produce an equal number

Table 2 Test of tetracycline treatment. Progeny from cross of $D$. virilis $w q \times D$. lummei $\delta$. See text for details

\begin{tabular}{llllll}
\hline Treatment & Eyeless & $\begin{array}{l}\text { Winglength } \\
\text { asymmetry }\end{array}$ & $\begin{array}{l}\text { Wing vein } \\
\text { anomaly }\end{array}$ & Normal & Total \\
\hline $\begin{array}{l}\text { Tetracycline } \\
\quad+\end{array}$ & 1 & 4 & & 34 & 49 \\
$\delta$ & 0 & 2 & 10 & 26 & 37 \\
No tetracycline & & & 9 & 221 & 271 \\
+ & 2 & 42 & 6 & 164 & 199 \\
$\delta$ & 1 & 28 & 6 & & \\
\hline
\end{tabular}


Table 3 Frequency of developmental anomalies among progeny of backcross females. The two classes of backcross mothers, $w q(\mathrm{LV}) \mathrm{V}$ and $+q(\mathrm{LV}) \mathrm{V}$, differ only in their $\mathrm{X}$ chromosome genotype. These females were crossed to $D$. lummei males. The genotype of the offspring is given below that of the mother. Note that the cytoplasm is ultimately derived from $D$. lummei

\begin{tabular}{|c|c|c|c|c|c|}
\hline $\begin{array}{l}\text { Mother's } \\
\text { genotype }\end{array}$ & Eyeless & $\begin{array}{l}\text { Winglength } \\
\text { asymmetry }\end{array}$ & $\begin{array}{l}\text { Wing vein } \\
\text { anomaly }\end{array}$ & Normal & Total \\
\hline \multicolumn{6}{|l|}{$w q(\mathrm{LV}) \mathrm{V}$} \\
\hline$q(+)$ & 1 & 7 & 3 & 228 & 239 \\
\hline$\partial(w)$ & 1 & 2 & 4 & 174 & 181 \\
\hline \multicolumn{6}{|l|}{$+q(\mathrm{LV}) \mathrm{V}$} \\
\hline$q(+)$ & 2 & 4 & 3 & 224 & 233 \\
\hline$\delta(w)$ & 1 & 5 & 3 & 131 & 140 \\
\hline$\sigma(+)$ & 0 & 3 & 0 & 88 & 91 \\
\hline
\end{tabular}

of anomalous offspring (comparing daughters from two genotypes of mothers, $\chi^{2}=1 \cdot 11, \mathrm{df}=3$, $P>0.75$; comparing white sons from two genotypes of mothers, $\left.\chi^{2}=2 \cdot 29, \mathrm{df}=3, P>0 \cdot 50\right)$. One cannot, however, rule out the possibility that $\mathrm{X}$-linked loci far to the left of the $w$ locus are involved: although $D$. virilis and $D$. lummei are fixed for two large, overlapping inversion differences in the right end of the X, almost surely including the $w$ locus (see Gubenko and Evgen'ev, 1984), the left ends of the $\mathrm{X}$ are apparently homosequential (see Throckmorton, 1982, and references therein).

The fact that so few hybrid anomalies $(4 \cdot 4$ per cent) appear among the progeny of these backcross females suggests that more than one maternallyacting locus from $D$. virilis may be involved: if only one locus were involved approximately 14 per cent $(=0.5 \times 27$ per cent $)$ of these progeny would show an anomaly. If, however, two genes are involved, the expected percentage drops to 7 per cent $(=0.25 \times 27$ per cent $)$, closer to the observed $4 \cdot 4$ per cent.

The results obtained thus far-hybrid anomalies are non-reciprocal, and involve a maternal effect; the maternally-acting genes are likely to be autosomal; and the alleles from $D$. virilis are recessive-are strikingly similar to the genetics of microchromosome loss in $D$. virilis-D. lummei hybrids. Sokolov $(1948,1959)$ showed that the tiny D. lummei sixth chromosome is frequently eliminated from $D$. virilis female $\times D$. lummei male hybrids, but not from hybrids from the reciprocal cross. He demonstrated that this non-reciprocality results from a maternal effect, not from some independent cytoplasmic agent. Evgen'ev (1973) and Evgen'ev and Sidorova (1976) further showed that loss of the microchromosome involves maternally-acting "mitosis genes" from $D$. virilis on the second and fourth chromosomes (mitotic division in early development is under the control of maternally-acting genes). These $D$. virilis alleles are nearly completely recessive (Evgen'ev and Sidorova, 1976). These similarities suggest that the hybrid developmental anomalies may result from loss of the $D$. lummei microchromosome during development.

To test this possibility, I performed two additional experiments. First, Evgen'ev and Sidorova (1976) showed that elimination of the D. lummei microchromosome in $D$. virilis cytoplasm is temperature-sensitive: loss is far more frequent at lower temperatures. I thus crossed $D$. virilis $w$ females to $D$. lummei males at $18^{\circ} \mathrm{C}$ vs. $22^{\circ} \mathrm{C}$. Low temperature treatment greatly increases the frequency of developmental anomalies (table 4 , pooling homogeneous female and male data, $\chi^{2}=$ $110 \cdot 84, \mathrm{df}=1, P<0 \cdot 0001$; because anomalies were so common at $18^{\circ} \mathrm{C}$, producing many hybrids carrying multiple anomalies, flies were simply scored as "anomalous" or "normal").

Next, I determined whether hybrids who had lost the $D$. lummei microchromosome were more likely to show anomalies than hybrids who had not lost a microchromosome. Loss of the D. lummei microchromosome is easily detected among

Table 4 Effect of temperature on frequency of hybrid developmental anomalies. All flies result from the cross $D$. virilis $w \% \times D$. lummei $\delta$

\begin{tabular}{lccl}
\hline Temperature & Anomalous & Normal & $\begin{array}{l}\text { Per cent } \\
\text { anomalous }\end{array}$ \\
\hline $18^{\circ} \mathrm{C}$ & & & \\
$\wp$ & 101 & 53 & $65 \cdot 6$ \\
$\delta^{\circ}$ & 41 & 22 & $65 \cdot 1$ \\
$22^{\circ} \mathrm{C}$ & & & \\
$\wp$ & 63 & 189 & $25 \cdot 0$ \\
$\delta^{\circ}$ & 48 & 146 & $24 \cdot 7$ \\
\hline
\end{tabular}


hybrids by crossing $D$. virilis $g l / g l$ females to $D$. lummei wild-type males: loss results in flies expressing the recessive $g l$ allele from $D$. virilis. If the microchromosome is lost in very early cleavages, hybrids possess one or both glossy eyes; loss later in development results in hybrids who are mosaics showing progressively less of the glossy phenotype (Evgen'ev and Sidorova, 1976).

It is important to note that even if anomalies occur only when the $D$. lummei microchromosome is lost, the observed association between the appearance of the anomalies and of the glossy phenotype will be imperfect for two reasons. First, one cannot detect all anomalies (many may be too subtle or may be internal (Sokolov, 1959)). Second, one cannot detect cases where the $D$. lummei sixth is lost late in development in small patches of tissue, causing localized anomalies, but is not lost in the eye tissue. The first "error" will result in flies showing the glossy phenotype but no anomalies, while the second "error" will result in flies showing no glossy, but suffering from slight anomalies.

Control crosses show that microchromosome loss is very rare within the species $D$. virilis (table 5 , top). However, in the $D$. virilis pe; $g l$ female $\times$ $D$. lummei male hybridization, two-thirds of the hybrids showed loss of the D. lummei microchromosome (table 5). Flies with anomalies showed microchromosome loss far more often than did non-anomalous flies (table $5 ; \chi^{2}=34 \cdot 09, \mathrm{df}=2$, $P<0 \cdot 0001$ ). Indeed, a third of all anomalous flies showed an extreme ("whole-eye") glossy phenotype, and half were glossy mosaics, while only 17 per cent showed no loss of the $D$. lummei sixth. Among flies showing no anomalies, only 9 per cent showed an extreme glossy, while 49 per cent were glossy mosaics, and 41 per cent showed no loss of the sixth. Thus loss of the $D$. lummei microchromosome in development is strongly associated with the appearance of hybrio anomalies. Loss in early development (producing an extreme glossy phenotype) renders hybrids particularly prone to anomalies. These results strongly suggest that the hybrid anomalies result from elimination of the $D$. lummei microchromosome from hybrids.

Analogous crosses using the $D$. virilis markers $v a$ (II), tb (III), cd (IV), and pe (V) showed that the $D$. lummei major chromosomes are very rarely lost from hybrids (0/306 varnished mosaics, $0 / 351$ cardinal mosaics, $0 / 351$ peach mosaics were recovered; although $3 / 351$ possible tiny bristle mosaics were recovered, these "mosaics" arose at far too low a frequency to account for the anomalies). The extreme rarity of major chromosome loss is not surprising as loss would probably be lethal except in very small patches of tissue.

To determine if anomalies arise when $D$. virilis hybridizes with other species in the virilis group, I crossed $D$. virilis females to $D$. novamexicana, $D$. americana, and $D$. texana males. As table 5 shows, less than 4 per cent of $D$. virilis- $D$. novamexicana hybrids show any discernible anomaly. All these anomalies were very slight, usually involving subtle disturbances in wing venation. Loss of the $D$. novamexicana microchromosome is also fairly rare ( $<3$ per cent); loss never occurred early enough in development to produce an entirely glossy eye. Anomalous flies are significantly more likely than normal flies to be glossy mosaics $\left(\chi^{2}\right.$ with continuity correction $=5.92, \mathrm{df}=$ $1, P<0 \cdot 02$ ), although the anomalies and mosaicism are too rare to allow much confidence in any statistical association. It is interesting, however, that in a hybridization in which microchromosome loss is rare and occurs late in development, developmental anomalies are also rare and, when they occur, are relatively subtle. Anomalies were extremely rare in the $D$. virilis $\times D$. americana and

Table 5 Frequency of loss of microchromosome among anomalous vs. normal-appearing individuals in various crosses. Loss of microchromosome is indicated by appearance of glossy phenotype among hybrids

\begin{tabular}{|c|c|c|c|c|}
\hline & & t-eye & $g l$ mosaic & $g l$ whole eye \\
\hline \multirow[t]{2}{*}{ D. virilis pe; $g l q \times D$. virilis Pasadena $\hat{o}$} & Anomalous & 0 & 0 & 0 \\
\hline & Normal & 154 & 0 & 1 \\
\hline \multirow[t]{2}{*}{ D. virilis pe; $g l \propto \times D$. lummei Finland $\delta$} & Anomalous & 16 & 48 & 31 \\
\hline & Normal & 93 & 111 & 21 \\
\hline \multirow[t]{2}{*}{ D. virilis pe; $g l \propto \times D$. novamexicana San Antonio o } & Anomalous & 8 & 2 & 0 \\
\hline & Normal & 242 & 5 & 0 \\
\hline \multirow[t]{2}{*}{ D. virilis pe; $g l \propto \times D$. americana Red Cloud $\delta$} & Anomalous & 1 & 0 & 0 \\
\hline & Normal & 63 & 0 & 0 \\
\hline \multirow[t]{2}{*}{ D. virilis pe; $g l q \times D$. texana Morrilton $\delta^{\star}$} & Anomalous & 1 & 0 & 0 \\
\hline & Normal & 165 & 0 & 0 \\
\hline
\end{tabular}


$D$. virilis $\times D$. texana hybridizations. No glossy mosaics were observed.

Thus there is strong, but indirect, evidence suggesting that anomalies result from microchromosome loss. Unfortunately, it would seem prohibitively difficult to obtain more direct evidence on this point. Although one could examine mitotic preparations of anomalous vs. control normal tissue for absence of one of the very small dot chromosomes, this approach seems unlikely to succeed: because anomalies are usually seen in adult eye and wing tissues - which do not polytenizeone has little hope of unambiguously scoring the presence or absence of the tiny $D$. lummei homologue. While, in principle, molecular tests of anomalous vs. normal tissue might be more likely to succeed, no 6-linked electrophoretic markers are known in $D$. virilis; similarly, no DNA probes for 6-linked sequences are available. Moreover, such a marker or probe would be useful only if $D$. virilis and $D$. lummei showed fixed differences at the relevant locus.

\section{DISCUSSION}

It appears that developmental anomalies among $D$. virilis-D. lummei hybrids are caused by loss of the D. lummei microchromosome during development. The evidence for this is: (1) both microchromosome loss and the anomalies occur non-reciprocally $(D$. virilis female $\times D$. lummei male only) and involve a maternal effect; (2) in both cases the maternally-acting alleles from $D$. virilis act as recessives; (3) the maternal genes causing microchromosome loss are autosomal; the maternal genes causing the anomalies appear to be autosomal; (4) the incidence of both microchromosome elimination and production of anomalies increases with cold temperature treatment; (5) there is a strong association between microchromosome loss (as indicated by glossy phenotype) and the appearance of anomalies; (6) loss of the microchromosome early in development is particularly strongly correlated with appearance of the anomalies.

Although they do not suggest that the hybrid anomalies result from loss of a microchromosome, Heikkinen and Lummei (1989) claim in their recent note that the anomalies involve maternally-acting genes on chromosomes 2 and 5 (no data are presented). Loss of the $D$. lummei microchromosome apparently involves maternally-acting genes on chromosomes 2 and 4 (mothers who are $D$. virilis homozygotes for either chromosome can produce anomalous offspring (Evgen'ev and Sidorova, 1976)). Because these experiments employed different strains, and probably different temperature regimes, it is difficult to compare these results (i.e., as Evgen'ev and Sidorova (1976) showed, whether a chromosome appears to have a large or smaller effect on chromosome loss depends on the temperature regime). It would be interesting to simultaneously analyze the genetic basis of microchromosome loss and anomaly production using the same strains under identical environmental conditions. The results of this paper suggest that the same chromosomes showing a maternal effect on microchromosome loss early in development will show a large maternal effect on anomaly production.

Loss of a homolog may be uncommon in animal hybridizations: I am aware of no other cases of chromosome elimination in the literature. It is thus perhaps not surprising that the anomalies do not obey two of the known "rules" of speciation: the anomalies do not affect males more than females ("Haldane's rule"), nor involve a large effect of the X chromosome (Coyne and Orr, 1989).

The anomalies do, however, involve a maternal effect: maternal effects on postzygotic isolation are quite common in Drosophila (Orr, 1989). Although the specific mechanisms involved are usually unknown (but see Kinsey [1967]), the basis of the maternal effect seems clear in this case. Mitosis in early development is under maternal control; because at least two maternally-acting "mitosis genes" have diverged between $D$. virilis and $D$. lummei, the late-replicating $D$. lummei microchromosome is frequently eliminated during mitosis when present in cytoplasm conditioned by these homozygous D. virilis alleles (Evgen'ev, 1973; Evgen'ev and Sidorova, 1976; Evgen'ev and Gubenko, 1977). Anomalies apparently arise because the resulting tissues are haplo-6. It is interesting to note that most of the abnormalities observed among the $D$. virilis $-D$. lummei hybrids resemble mutations that are known on the $D$. virilis microchromosome or on the homologous $D$. melanogaster microchromosome (Sturtevant and Novitski, 1941): eyeless (ey), cubitus interruptus $(c i=\mathrm{Gap}[G p])$, and abdomen rotatum (ar) (see Gubenko and Evgen'ev, 1984; Lindsley and Grell, 1968). Although these similarities may be coincidental, this phenotypic resemblance might be expected if these mutants are loss-of-function mutations.

It is important to note that while the proximal cause of the hybrid anomalies may be "chromosomal", the ultimate cause of the anomalies is 
nonetheless "genic". That is, chromosome loss results from the inability of maternally-acting genes in $D$. virilis to interact properly with certain dominant, zygotically-acting gene(s) in D. lummei. Evgen'ev and Gubenko (1977) present evidence suggesting that the latter factor(s) is (are) on the D. lummei microchromosome itself. Thus while the manifestation of hybrid incompatibility is rather unusual (chromosome loss), the cause of this breakdown is quite ordinary: a disruption of normal epistatic interactions between genes (Muller, 1942a). Dobzhansky (1937) has similarly argued that in most (if not all) animal hybridizations, the failure of chromosomes to pair properly in meiosis reflects a genic, not a chromosomal, incompatibility. In short, one cannot attribute unusual chromosome behaviour in hybridswhether mitotic or meiotic-to structural differences in chromosomes between species, as chromosome behaviour is under the control of genes which may fail to properly interact in species hybrids.

Although mere speculation, maternal control of mitosis in early development could explain the frequent observation of maternal effects on hybrid viability in Drosophila (e.g., Kaufmann, 1940): hybrid embryonic inviability may result from a breakdown in mitosis (not necessarily involving chromosome elimination). Similarly, frequent maternal effects on hybrid fertility (e.g., Orr, 1989) are not unexpected as the primordial germ cells are set aside early in Drosophila development and so are under strong maternal control (Mahowald et al., 1976). It is important to note, however, that even if correct, one must explain why these maternally-acting mitosis/meiosis genes are especially likely to diverge between species, and thus be involved in postzygotic isolation.

Last, these results show that some caution is needed when interpreting the evolutionary significance of unusual morphologies among the hybrids of sibling species. Anomalies in a character obviously show that the similar phenotypes of the two species have different genetic bases, i.e., there has been cryptic divergence in the genes underlying this character. Similarly, anomalies in several characters among hybrids (e.g., missing bristles, heavier trident, and eye-reduction in $D$. melanogaster- $D$. simulans partial hybrids) are usually taken as evidence that there has been extensive genetic divergence (e.g., Muller, 1942b), i.e., so many gene substitutions have occurred between these sibling species that many different characters are affected among hybrids.

However, as the present results show, an alternative explanation is possible: disturbances in many characters may have a common genetic cause, and a simple genetic basis. Thus the fact that $D$. virilis $-D$. lummei hybrids show unusual eye, wing length, wing vein, abdomen, and bristle phenotypes does not demonstrate that each of these characters has a different, somewhat incompatible, genetic basis in these closely related species. Instead, these unusual phenotypes are likely symptoms of a single problem (microchromosome loss), which almost certainly has a simple genetic basis.

To determine whether hybrid anomalies result from cryptic divergence of many independent characters under stabilizing selection or are pleiotropic effects of some single genic incompatibility (not necessarily involving chromosome loss) one must perform genetic analysis, e.g., hybrid backcross analysis. The former hypothesis allows that a particular chromosome substitution can affect different hybrid characters differently. The latter hypothesis predicts that a substitution affects all anomalies similarly as all share a common genetic cause. Unfortunately, these two possibilities-with their very different evolutionary implications-have been disentangled in few, if any, other animal hybridizations.

Acknowledgments I thank Kevin Matthews and Jim Shorts for their invaluable conversation; this work would not have been possible without their constant input and inspiration. I also thank M. Evgen'ev for his helpful correspondence, and J. Coyne for his valuable comments on this manuscript. This work was supported by training grant GM 07197 from the National Institute of General and Medical Sciences of the National Institutes of Health to the University of Chicago, and by National Institutes of Health grant GM 38462 to J. Coyne.

\section{REFERENCES}

ALEXANDER, M. L. 1976. The genetics of Drosophila virilis. In Ashburner, M. and Novitski, E. (eds) The Genetics and Biology of Drosophila, vol. 1c, Academic Press, Inc., London.

COYNE, J. A. AND ORR, H. A. 1989. Two rules of speciation. In Otte, D. and Endler, J. A. (eds) Speciation and its consequences, Sinauer Associates, Sunderland, MA, pp. 180-207.

DObZHANSKy, T. 1937. Genetics and the Origin of Species. Columbia Univ. Press, New York.

EVGEN'EV, M. B. 1973. Genetic regulation of mitosis in interspecific hybrids of Drosophila. I. Maternal effect on chromosome segregation and elimination. Genetica, 9, 9299.

EVGEN'EV, M. B. AND SIDOROVA, N. V. 1976. Genetic regulation of chromosome behavior in interspecific hybrids of Drosophila. Theor. Appl. Gen., 48, 55-61. 
EVGEN'EV, M. B. AND GUBENKO, I. S. 1977. Genetic regulation of the replication pattern of polytene chromosomes in interspecific hybrids of Drosophila. Chromosoma, 63, 89100.

GUBENKO, I. S. AND EVGEN'EV, M. B. 1984. Cytological and linkage maps of Drosophila virilis chromosomes. Genetica, $65,127-139$.

HEIKKINEN, E. AND LUMME, J. 1989. Maternally determined eye syndrome in interspecific hybrids. European Drosophila Research Conference (Abstracts), 11, 22.

HOFFMAN, A. A. AND TURELLI, M. 1988. Unidirectional incompatibility in Drosophila simulans: inheritance, geographic variation and fitness effects. Genetics, 119, 435-444.

KAUFMANN, B. P. 1940. The nature of hybrid sterility-abnormal development in eggs of hybrids between Drosophila miranda and Drosophila pseudoobscura. J. Morphol., 66, 197-213.

KINSEY, J. D. 1967. Studies on an embryonic lethal hybrid in Drosophila. J. Embryol. Exp. Morphol, 17, 405-423.

LINDSLEY, D. L. AND GRELL, E. H. 1968. Genetic Variations of Drosophila melanogaster. Carnegie Inst. Wash. Publ. 627.

MAHOWALD, A. P., ILLMENSEE, K. AND TURNER, F. R. 1976. Interspecific transplantation of polar plasm between Drosophila embryos. Journal of Cell Biology, 70, 358-373.

MUL.LER, H. J. 1942a. Isolating mechanisms, evolution and temperature. Biol. Symposia, 6, 71-125.
MULlER, H. J. 1942b. Recessive genes causing interspecific sterility and other disarmonies between Drosophila melanogaster and simulans. Genetics, 27, 157.

ORR, H. A. 1989. Genetics of sterility in hybrids between two subspecies of Drosophila. Evolution, 43, 180-189.

ORR, H. A. AND COYNE, J. A. 1989. The genetics of postzygotic isolation in the Drosophila virilis group. Genetics, 121, 527-537.

SOKOLOV, N. N. 1948. Elimination of chromosomes in interspecific hybrids of Drosophila and the problems of distant hybrids. Dokladi Academii Nauk, 59, 163-166 (In Russian).

SOKOLOV, N. N. 1959. The interaction of nucleus and cytoplasm in distant hybrids. Moscow: Izdatelstvo Academii Nauk, 1-148 (In Russian).

Sturtevant, A. H. 1920. Genetic studies on Drosophila simulans. I. Introduction. Hybrids with Drosophila melanogaster. Genetics, 5, 488-500.

SturtevanT, A. H. 1929. The Genetics of Drosophila simulans. Carnegie Inst. Wash. Publ. 399, pp. 1-62.

STURTEVANT, A. H. AND NOVITSKI, E. 1941. The homologies of the chromosome elements in the genus Drosophila. Genetics, 26, 517-541.

THROCKMORTON, L. H. 1982. The virilis species group. In Ashburner, M., Carson, H. L. and Thompson, J. N. Jr. (eds) The Genetics and Biology of Drosophila, vol. 3b, Academic Press, London, pp. 227-296. 